Please do not remove this page

RMIT

UNIVERSITY

\title{
A hybrid reconstruction algorithm for 3-D ionospheric tomography
}

Wen, Debao; Yuan, Yunbin; Ou, Jikun; Zhang, Kefei; Liu, Kai

https://researchrepository.rmit.edu.au/esploro/outputs/9921861873901341/filesAndLinks?institution=61RMIT_INST\&index=null

Wen, D., Yuan, Y., Ou, J., Zhang, K., \& Liu, K. (2008). A hybrid reconstruction algorithm for 3-D ionospheric tomography. IEEE Transactions on Geoscience and Remote Sensing, 46(6), 1733-1739.

https://doi.org/10.1109/TGRS.2008.916466

Published Version: https://doi.org/10.1109/TGRS.2008.916466

Repository homepage: https://researchrepository.rmit.edu.au

(c) 2008 IEEE. Personal use of this material is permitted. However, permission to reprint/republish this material for advertising or promotional purposes or for creating new collective works for resale or redistribution to servers or lists, or to reuse any copyrighted component of this work in other works must be obtained from the IEEE.

Downloaded On 2023/04/26 12:21:04 +1000 


\title{
A Hybrid Reconstruction Algorithm for 3-D Ionospheric Tomography
}

\author{
Debao Wen, Yunbin Yuan, Jikun Ou, Kefei Zhang, and Kai Liu
}

\begin{abstract}
In this paper, a hybrid reconstruction algorithm (HRA) is presented to solve the ill-posed inverse problem associated with 3-D ionospheric stochastic tomography. In this new method, the ionospheric electron density (IED) can be inverted by using two steps. First, a truncated singular value decomposition (TSVD) method, whose value is independent on any initial estimation, is used to resolve the ill-posed problem of the tomography system. Second, taking into account the "approximation" of its solution, an iterative improvement process of the solution is then implemented by utilizing the conventional algebraic reconstruction algorithm (ART). The HRA, therefore, offers a more reasonable approach to choose an initial approximate for the ART and to improve the quality of the final reconstructed image. A simulated experiment demonstrates that the HRA method is superior to the TSVD or the ART alone for the tomographic inversion of IED. Finally, the HRA is used to perform GPS-based tomographic reconstruction of the IED at mid- and low-latitude regions.
\end{abstract}

Index Terms-Inverse problem, ionosphere, tomography.

\section{INTRODUCTION}

$\mathbf{T}$ HE IONOSPHERE is the atmosphere at the altitudes from 60 to $1000 \mathrm{~km}$ above the Earth's surface. Ionospheric total electron content (TEC), which is the line integral of ionospheric electron density (IED) along the ray path from a satellite to a ground receiver, produces a time delay in a satellite radio wave when it traverses through the ionosphere. Thus, the accuracy of satellite navigation and positioning is affected by the electron density distribution in the ionosphere. For this reason, satellite systems, such as the global positioning system (GPS), usually transmit two frequencies and make use of the dispersive nature of the ionosphere to correct for the delay effects. When the radio signals from an orbiting satellite are received by a ground receiver, the ionospheric TEC can be obtained and used to study the variations of the horizontal structure of the ionosphere.

Manuscript received December 15, 2006; revised August 22, 2007. This work was supported by the National Science Fund for Distinguished Young Scholars of China under Grant 40625013, by the National Natural Science Foundation of China under Grant 40674012, and by the 863 program under Grant 2007AA12Z311.

D. Wen is with the Institute of Geodesy and Geophysics, Chinese Academy of Sciences, Wuhan 430077, China, with the Changsha University of Science and Technology, Changsha 410076, China, and also with the Graduate School of the Chinese Academy of Sciences, Beijing 100049, China.

Y. Yuan and J. Ou are with the Institute of Geodesy and Geophysics, Chinese Academy of Sciences, Wuhan 430077, China.

$\mathrm{K}$. Zhang is with the School of Mathematical and Geospatial Sciences, Royal Melbourne Institute of Technology, Melbourne, Vic. 3001, Australia.

K. Liu is with Jinan Geotechnical Investigation and Surveying Institute, Jinan 250013, China (e-mail: Pingwennews2161@126.com).

Color versions of one or more of the figures in this paper are available online at http://ieeexplore.ieee.org.

Digital Object Identifier 10.1109/TGRS.2008.916466
However, the vertical variation of the ionospheric structure cannot be easily recovered since the conventional 2-D models used for ionospheric TEC determination cannot be directly applied to derive the vertical electron density distributions along a ray path. This limits the temporal and spatial resolution of the modeled results and the correction accuracy of ionospheric delay. For academic or practical applications, it is necessary to reconstruct the IED vertical distributions with an effective and feasible method or technique.

Since Austen et al. [1] first demonstrated the possibility of reconstructing IED by using satellite radio-based tomography, the tomographic reconstruction technique has become a popular and successful means of studying the IED distribution. From then on, various investigations [2]-[5] and experiments [6], [7] have been carried out. In these studies, the observation data received by several ground stations are usually obtained from the Navy Navigation Satellite System (NNSS). Although these investigations are helpful for studying the vertical variations of ionospheric structure, only a 2-D ionospheric image along a fixed longitude chain can be reconstructed [8]. The advent of GPS has opened a new avenue for monitoring and studying ionospheric activities due to its advantageous characteristics, including its high precision and near-real-time. In recent years, some GPS-based tomographic investigations have been carried out [9]-[22]. Research results show that GPS-based tomography is suitable for the reconstruction of IED distributions in a 3-D mode, and it is advantageous to overcome the limitations associated with 2-D models. At present, GPS-based tomographic reconstruction of IED has become a focus of research in the fields of ionospheric physics and space geodesy since it can reconstruct the ionospheric image in 3-D mode. However, the existence of some limitations (e.g., the absence of horizontal ray paths, the limited number of ground stations, and finite receiving apertures) often makes the GPS-based ionospheric tomography an ill-posed problem.

To resolve this problem, numerous algorithms have been presented in recent years. As a whole, these algorithms can be divided into two categories: iterative reconstruction algorithms and noniterative reconstruction algorithms. The algebraic reconstruction algorithm (ART) is a conventional iterative method due to its simplicity in computation. It resolves the illposed problem by incorporating some prior information into each pixel in an ionospheric tomography system. For those pixels without any ray path traversing them, ART compensates for the incomplete data by using a good initial approximate at the overall distribution. This means that the ART is sensitive to the initial approximate. Among the noniterative reconstruction algorithms, the truncated singular value decomposition (TSVD) 
is a popular method for solving the ill-posed inverse problem in the ionospheric tomography system. An advantage of the TSVD is that the reconstruction quality does not depend on any initial approximate because its solution can be calculated directly from TEC data without any initial estimates. However, its solution is usually an approximation of the real solution due to the limitations of the insufficient number of available observations and to the impacts of discretized errors and observation noises.

Accounting for the disadvantages of these tomographic inversion algorithms, this paper presents a hybrid reconstruction algorithm (HRA) in order to improve the inversion accuracy of IED. This algorithm is the combination of the TSVD and ART. Numerical simulation experiment demonstrates that the HRA is superior to the TSVD or the ART alone in performing ionospheric tomography. Finally, the HRA method is applied to perform GPS-based tomographic reconstruction of the IED by using the GPS observations in China region.

\section{TOMOGRAPHIC FORMULATION}

To reconstruct the 3-D images of IED distributions, the line integral of the ionospheric electron density along ray paths traversing the imaged region should first be derived from dualfrequency GPS data. The line integral of IED is the ionospheric slant TEC (STEC), which can be mathematically represented as

$$
\mathrm{STEC}=\int_{p} N(s) d s
$$

where $N(s)$ represents the IED, $p$ is the ray propagation path between a satellite and a receiver, STEC is the input data in the inverse process of IED. The derivation of STEC is commonly made using the pseudoranges and phase measurements of the dual-frequency GPS signals. Since GPS phase measurements are less affected by multipath effects, the accuracy of the STEC derived from differential phases $\left(\mathrm{STEC}_{\mathrm{ph}}\right)$ is higher than that of the STEC derived from differential pseudoranges $\left(\mathrm{STEC}_{\mathrm{co}}\right)$. Because of the ambiguity in GPS phase measurements, $\mathrm{STEC}_{\mathrm{ph}}$ only provides the relative changes of ionospheric TEC. On the other hand, although P-code pseudoranges are sensitive to multipath effects, STEC $_{\text {co }}$ values are absolute. Taking into account these facts, an absolute STEC with improved accuracy may be obtained by introducing an extra term $B_{L}$. It can be formulated as follows [14]:

$$
\mathrm{STEC}=\mathrm{STEC}_{\mathrm{ph}}+B_{L} .
$$

If $N$ measurements are obtained during a satellite pass, $B_{L}$ can be modeled by the following equation:

$$
B_{L}=\sqrt{\sum_{i=1}^{N}\left(\mathrm{STEC}_{\mathrm{co}_{\mathrm{i}}}-\mathrm{STEC}_{\mathrm{ph}_{i}}\right)^{2} / N} .
$$

According to the discrete inverse theory, an imaged region is usually divided into a number of small pixels. Within each pixel, the electron density can be assumed constant in a selected reference frame during a specific time period. Thus, the STEC along the ray path can be represented as a finite sum of shortest integrals along different segments of the ray path. The reconstruction process then centers on solving the following system of equations:

$$
\mathrm{STEC}_{\mathrm{i}}=\sum_{j=1}^{n} A_{i j} x_{j}+e_{i} .
$$

Equation (2) can be generally written in a simple matrix notation as

$$
y_{m \times 1}=A_{m \times n} x_{n \times 1}+e_{m \times 1}
$$

where $n$ is the number of pixels in the image region, $m$ is the number of TEC measurements, $y$ is a column vector of the $m$ known TEC measurements, $A$ is an $m \times n$ matrix with $A_{i j}$ being the length of ray $i$ traversing pixel $j, x$ is a column vector consisting of all the unknown electron densities in all the pixels, and $e$ is a column vector associated with the discretization errors and measurement noises.

\section{Hybrid Reconstruction Algorithm}

In an ionospheric tomography system, matrix $A$ is usually rank deficient. This means that only a subset of the $m$ equations is linearly independent. Moreover, the number of independent equations is generally less than the number of the unknown parameters. Therefore, the tomographic reconstruction of the IED is an underdetermined inverse problem. In this case, the singular value decomposition (SVD) of the matrix $A$ allows us to remove the linearly dependent equations. After performing the SVD, an $m \times n$ matrix $A$ can be written as

$$
A=U D V^{T}
$$

where $U$ is an $m \times L$ column-orthogonal matrix, $V$ is an $n \times L$ orthogonal matrix of eigenvectors, $D$ is an $L \times L$ diagonal matrix whose elements are positive and sorted in a decreasing order, and $L$ is the number of independent equations. After this decomposition, the least-squares solution for the system is given by

$$
x=V D^{-1} U^{T} y
$$

and the covariance of the solution can be represented as

$$
\operatorname{cov}(x)=\sigma^{2} V D^{-2} V^{T} .
$$

From (7), it is noted that the larger singular values included in the solution, the inverse result is more close to the true solution. On the other hand, since actual observation data contain various kinds of errors and noises, smaller singular values considerably amplify the numerical error in the inversion process [9]. Furthermore, they make the solutions severely perturbed, as seen from (8). Thus, it is necessary to perform a truncation for eliminating the impacts of the smaller singular values, and the TSVD of $A_{k}$ is defined as

$$
\mathrm{A}_{\mathrm{k}}=\sum_{i=1}^{k} U_{i} D_{i} V_{i}^{T}
$$


where $k<L$. This means that small nonzero $L-k$ singular values are truncated. Then the pseudoinverse $A_{k}^{+}$is written as

$$
\mathrm{A}_{\mathrm{k}}^{+}=\sum_{i=1}^{k} V_{i} D_{i}^{-1} U_{i}^{T}
$$

A reasonable truncation standard should be first determined for the application of (10). Various methods have been studied for choosing an approximate truncation value [23], [24]. For ionospheric tomography, a key issue is to choose an ideal truncation value such that the variance associated with the corresponding eigenvectors is physically meaningful. Assuming that the TEC measurement error is $10^{16} \mathrm{e} / \mathrm{m}^{2}$, a singular value (SV) truncation of $100 \mathrm{~km}$ would correspond to an uncertainty of $10^{11} \mathrm{e} / \mathrm{m}^{3}$ in a component of ionospheric electron density field. Given that the order of the error of the recovered IED is smaller than the above magnitude, the singular values, which are smaller than $100 \mathrm{~km}$, should be truncated [9]. One can then obtain the TSVD solution of (5) as follows:

$$
x_{k}=A_{k}^{+} y .
$$

The estimations obtained from (11) are then input as initial states required by the ART, as follows:

$$
x^{(0)}=x_{k} .
$$

Next, the reconstruction is performed with the following ART algorithm:

$$
x^{(k+1)}=x^{(k)}+\lambda_{k}\left(y_{i}-a_{i} x^{(k)}\right) .
$$

The column vector $\lambda_{k}$ consisting of all relaxation parameters is given as

$$
\lambda_{k}=\gamma \cdot a_{i}^{T} /\left(a_{i} a_{i}^{T}\right)
$$

where $a_{i}$ represents the $i$ th row vector in projection matrix $A$ and the relax parameter $\gamma$ is fixed in ionospheric tomography.

\section{Numerical Experiment Using Simulated Data}

Before the HRA is applied to the ionospheric reconstruction using actual TEC data, it is necessary to confirm its effectiveness by using simulated data. The simulated data are generated from the IRI2001 model. In this model, the ionospheric electron densities are smoothly variational and available from 50 to $2000 \mathrm{~km}$. The simulation procedure can be summarized in five steps.

1) In a selected reference frame and time period, the actual positions of GPS satellites and ground receivers are used to create the projection matrix $A$.

2) The IED at the center of each pixel is generated from the IRI2001 model and considered as the mean IED value of the corresponding pixel. The simulated time period is from 06:40 to 07:00 Universal time (UT) on February 14, 2002. The discrete density distribution is represented by $x_{\text {simu }}$. The simulated TEC values $y_{\text {simu }}$ without noise are then computed by using the following equation:

$$
y_{\text {simu }}=A \cdot x_{\text {simu }} \text {. }
$$
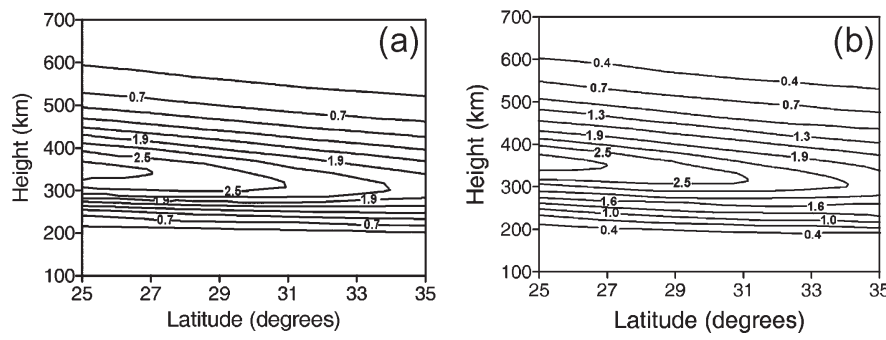

Fig. 1. Contour plots of modeled and reconstructed IED distributions at longitude $110^{\circ}$ E. (a) Modeled IED distribution with the IRI2001 model. (b) Reconstructed IED distribution with the HRA algorithm. The unit of IED is $10^{12} \mathrm{e} / \mathrm{m}^{3}$.
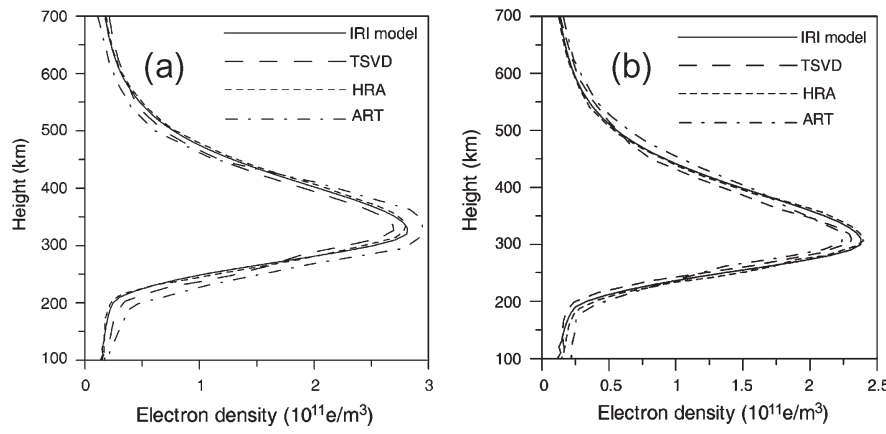

Fig. 2. Comparisons of IED profiles reconstructed from TSVD, ART, and HRA with those produced from the IRI2001 model at two geographic locations: $106^{\circ} \mathrm{E}, 28^{\circ} \mathrm{N}$ and $114^{\circ} \mathrm{E}, 32^{\circ} \mathrm{N}$. The IED is in unit of $10^{12} \mathrm{e} / \mathrm{m}^{3}$. (a) Comparisons between the reconstructed IED profile and the model at geographic locations $106^{\circ} \mathrm{E}, 28^{\circ} \mathrm{N}$. (b) Comparisons between the reconstructed IED profile and the model $114^{\circ} \mathrm{E}, 32^{\circ} \mathrm{N}$.

3) Practically, a small amount of random noise $e_{\text {simu }}$ should be added to the simulated TEC values $y_{\text {simu }}$. One can then obtain

$$
y_{\text {nois }}=y_{\text {simu }}+e_{\text {simu }} \text {. }
$$

The maximum noise used is $5 \%$ of the average of the simulated TEC values $y_{\text {simu }}$.

4) Using the above truncated standard, the TSVD is used to invert the IED from the simulated TEC with noise in (16). Its estimate $x_{e s t}$ is then obtained.

5) The estimate obtained from the TSVD is input as an initial state required by the ART. It is then improved by using (13) and (14).

Fig. 1(a) illustrates the electron density distribution at 07:00 UT on February 14, 2002, along $110^{\circ}$ E, which was obtained from the IRI2001 model. The reconstructed electron density distribution using the HRA is plotted in Fig. 1(b). The maximum absolute value of the reconstruction error is $1.67 \times$ $10^{10} \mathrm{e} / \mathrm{m}^{3}$, which is very small compared with the peak electron density of $2.8 \times 10^{12} \mathrm{e} / \mathrm{m}^{3}$. The root mean square (rms) is $1.3 \times 10^{10} \mathrm{e} / \mathrm{m}^{3}$. By comparing Fig. 1(a) and (b), it can be seen that the ionospheric structure is reconstructed very well. The results show that the electron density reconstruction is carried out with sufficiently high accuracy using the simulated data.

In Fig. 2, the modeled vertical IED distributions at two geographic positions- $106^{\circ} \mathrm{E}, 28^{\circ} \mathrm{N}$ and $114^{\circ} \mathrm{E}, 32^{\circ} \mathrm{N}$-are compared with those obtained from the TSVD, the ART, and the HRA methods. As shown in Fig. 2, the IED profiles obtained 
TABLE I

Statistics of the Reconstructed IED ERror Based on Three Algorithms ANd Simulated Data

\begin{tabular}{lccc}
\hline Reconstruction methods & ART & TSVD & HRA \\
\hline Maximum of the reconstructed IED errors $\left(10^{10} \mathrm{e} / \mathrm{m}^{3}\right)$ & 4.51 & 2.83 & 1.7 \\
Minimum of the reconstructed IED errors $\left(10^{10} \mathrm{e} / \mathrm{m}^{3}\right)$ & -6.76 & -4.15 & -1.07 \\
Average of the reconstructed IED errors $\left(10^{10} \mathrm{e} / \mathrm{m}^{3}\right)$ & 0.54 & 0.36 & 0.12 \\
Variance of the reconstructed IED $\left(10^{20}\left(\mathrm{e} / \mathrm{m}^{3}\right)^{2}\right)$ & 4.16 & 2.85 & 1.38 \\
\hline
\end{tabular}
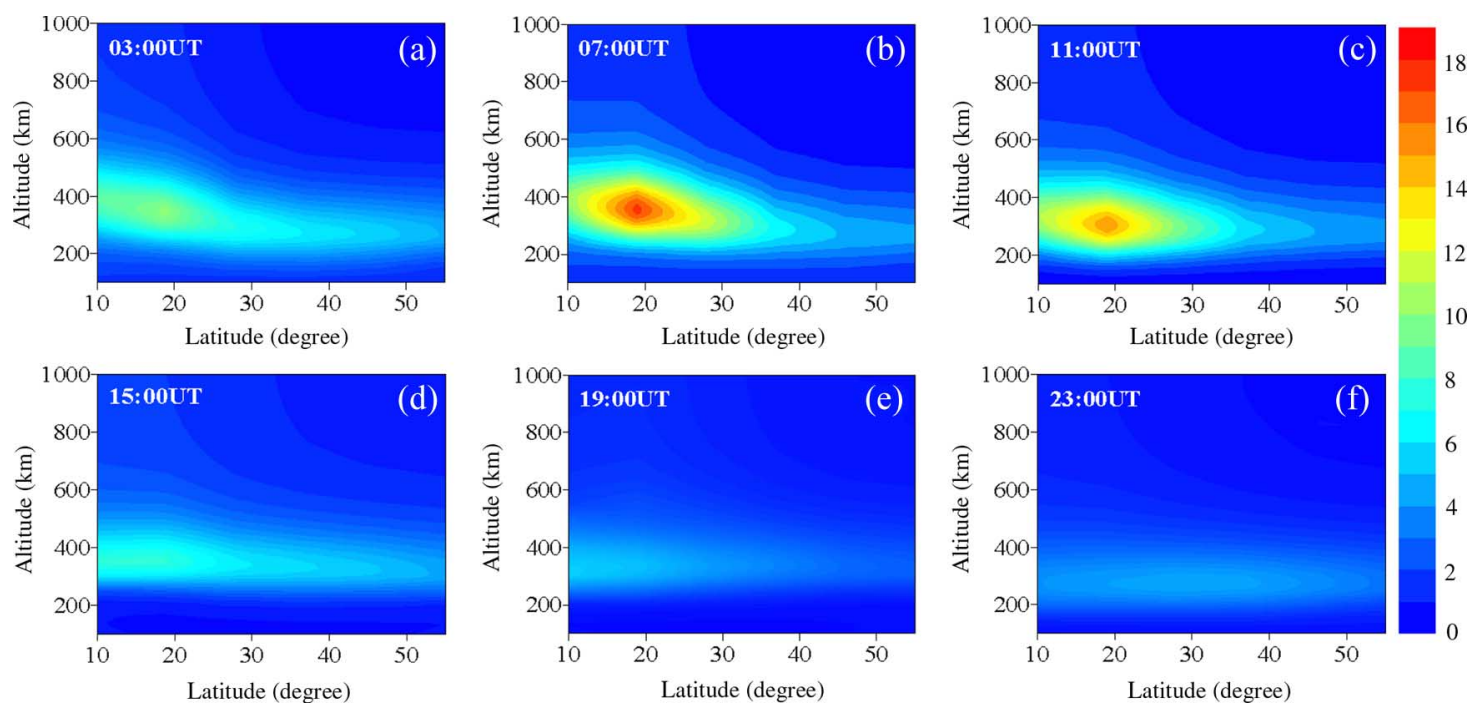

Fig. 3. Tomographic images monitored on August 15, 2003, derived from GPS data in China region. Recording time for each panel is provided at the top left corner of each panel. The unit of the color bar is $10^{11} \mathrm{el} / \mathrm{m}^{3}$.

from the HRA agree better with the modeled electron density profile than those obtained from the TSVD and the ART alone as a whole. Table I gives the statistics of the reconstruction error of all voxels in the reconstructed region. It shows that the reconstruction errors of the HRA are less than the TSVD or the ART alone. All the comparison results in Fig. 2 and Table I show that the HRA is superior to the TSVD or the ART alone in performing ionospheric tomography, and it can be applied to improve the quality of the reconstructed ionospheric image. The above tomographic results, therefore, verify the feasibility and the advantages of the HRA in performing ionospheric tomography.

\section{Applications to GPS-BASED IONOSPHERIC TOMOGRAPHY}

By using TEC data derived from GPS data, the HRA is applied to reconstruct the corresponding IED distribution. Taking the impact of the multipath effect into account, the elevation angle of GPS satellite at each observation station should be greater than $10^{\circ}$. The reconstruction algorithm includes the capability to automatically reject GPS data of elevation angle less than $10^{\circ}$ even if ray paths may intersect the image plane.

In this study, the dual-frequency GPS observation data from the Crustal Movement Observation Network of China
(CMONOC) on August 15, 2003, are applied to study the effects of hourly variations of the electron densities from 00:00 to 24:00 UT. Some sample examples of the hourly variations of the IED are shown in Fig. 3. Each snapshot is plotted with respect to latitude and height at the meridian plane of $114^{\circ} \mathrm{E}$. The IED is expressed in units of $10^{11} \mathrm{e} / \mathrm{m}^{3}$. By comparing Fig. 3(a)-(f), it can be seen that the peak height of the ionosphere gradually falls from $370 \mathrm{~km}$ to $300 \mathrm{~km}$ from 03:00 to 11:00 UT and then rises to $375 \mathrm{~km}$ at 19:00 UT. At 23:00 UT, the peak height also falls to $280 \mathrm{~km}$. This roughly reflects typical characteristics of the vertical variations of the ionospheric structure for the day over China. Fig. 3 also shows that an equatorial anomaly crest appeared at $18^{\circ} \mathrm{N}$ at 03:00 UT with a peak density of $1.0 \times 10^{12} \mathrm{e} / \mathrm{m}^{3}$. The tilting feature of the ionization crest can also be seen in this panel. As the time evolves, the crest is still seated at the same latitude position, but a characteristic anomaly core, which is defined as the region that has $80 \%$ to $90 \%$ of the crest value, is fully developed with a peak density of $1.81 \times 10^{12} \mathrm{e} / \mathrm{m}^{3}$ at 07:00 UT, and there is also a hint of the tilt of ionization crest. The gradient of the crest is reduced compared with the tilting feature shown at 03:00 UT. At 11:00 UT, the peak density of the anomaly crest is decreased to $1.21 \times 10^{12} \mathrm{e} / \mathrm{m}^{3}$, but it does not show any tilt. At 15:00 UT, the equatorial anomaly crest of the ionosphere has completely disappeared. 

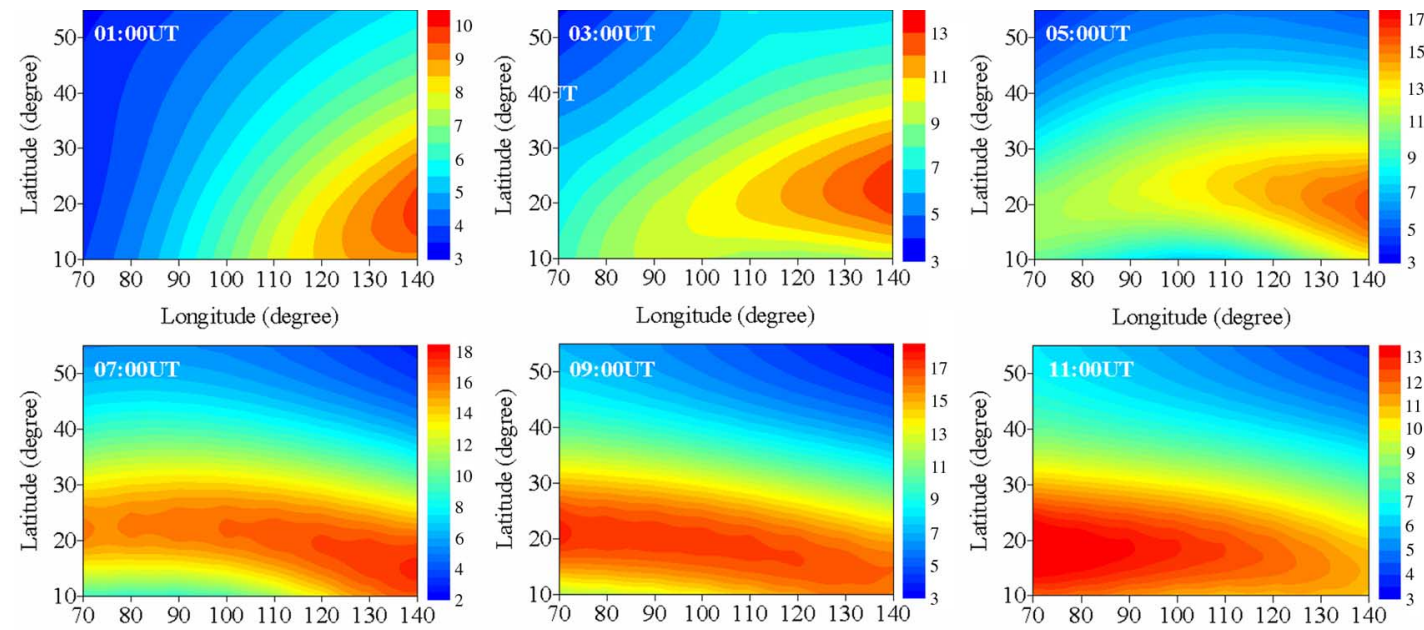

Longitude (degree)
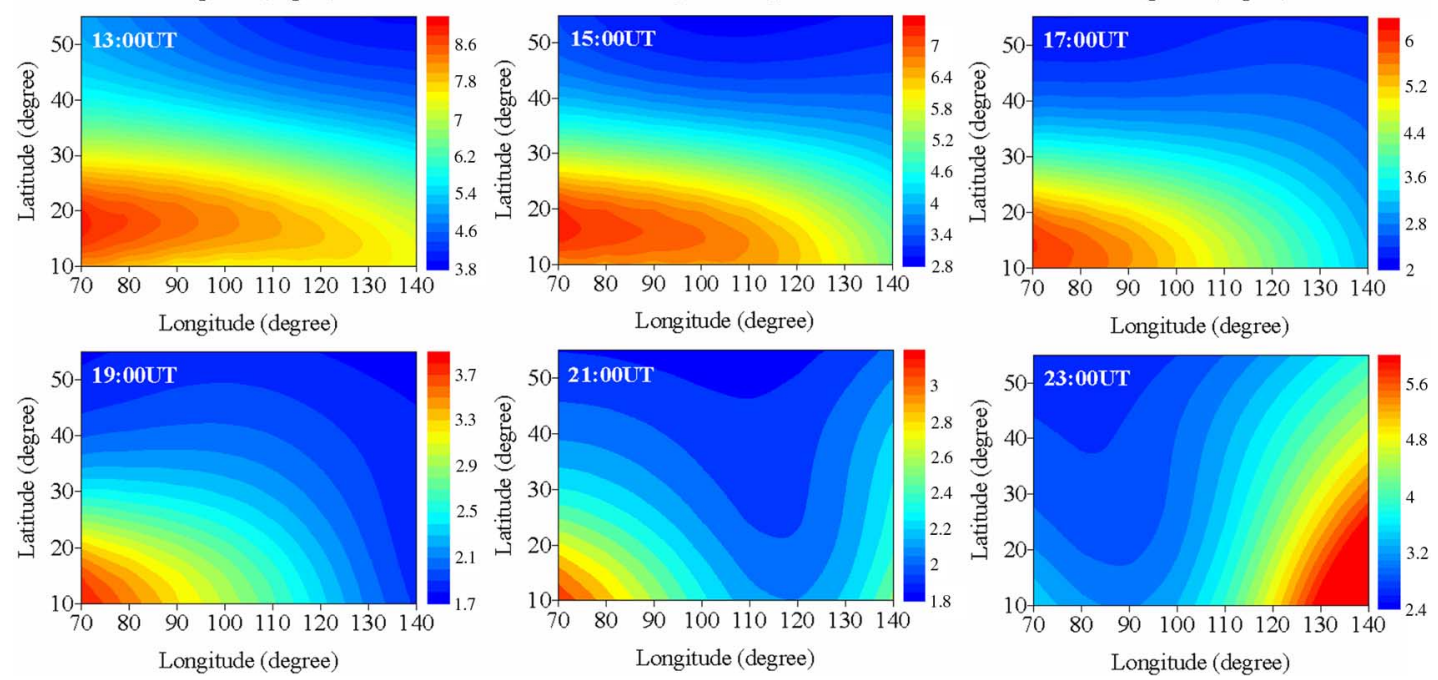

Fig. 4. Time-series variations of ionospheric electron density at the height of $350 \mathrm{~km}$ on August 15,2003 . The unit of the color bar is $10^{11} \mathrm{el} / \mathrm{m}^{3}$.
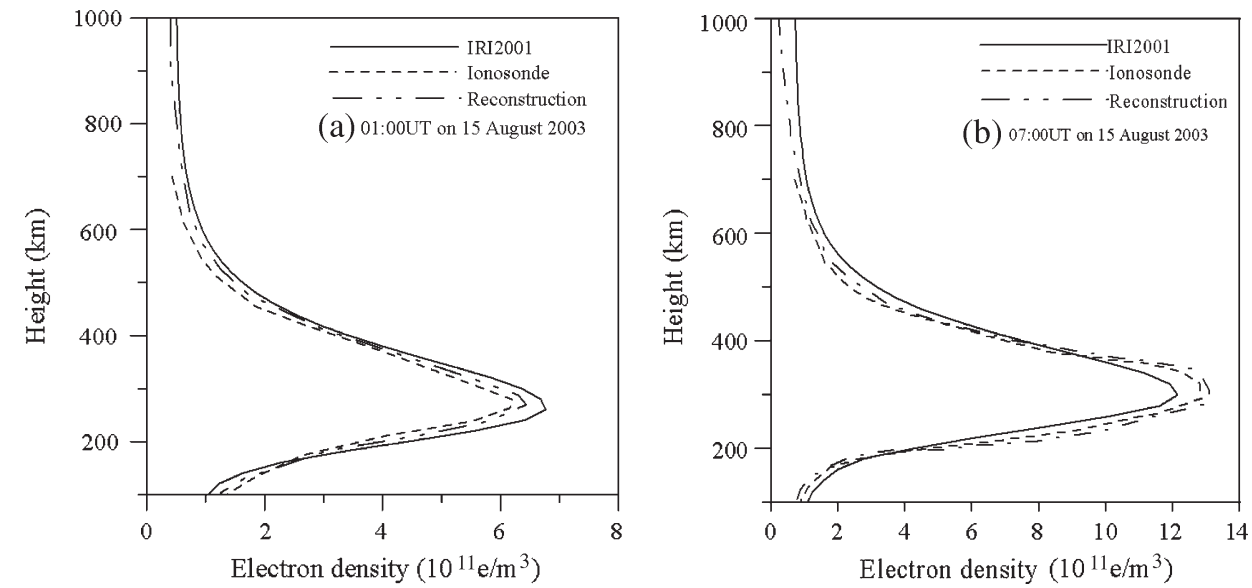

Fig. 5. Comparisons of the tomographically reconstructed electron density profiles with the corresponding density profiles measured by ionosonde station at Wuhan and those of IRI2001. (a) Comparison of IED profile at 01:00 UT. (b) Comparison of IED profile at 07:00 UT.

Fig. 4 shows that the time-series variation of IED as the latitude and longitude at the height of $350 \mathrm{~km}$ over China. From this figure, it can be seen that the inverted average IED values grow from small to large first and then fall from large to small, which is consistent with the Earth's rotation from west to east. This is also consistent with the normal change laws in daytime and night time over China as well as the fact that the characteristics of IED depends mainly on the dark solar activity. Furthermore, it can be seen that the values of IED in eastern China are larger than those in western China between 01:00 UT and 07:00 UT, and then the values of IED in western China are larger than those in eastern China between 09:00 UT 
and 21:00 UT as a whole. However, at 21:00 UT, the values of IED in eastern China begin to rise, and, at 23:00 UT, the values of IED recover the initial distribution (i.e., the values of IED in eastern China are larger than those in western China). Figs. 3 and 4 also show that there are larger differences between the characteristics of the IED in mid- and low-latitude areas, and the values of IED over northern China are smaller than those over southern China as a whole. This indicates a strong correlation of the variation of IED with latitude.

Fig. 5 provides independent comparisons of the reconstructed electron density profiles with those obtained from the IRI2001 model and the ionosonde data recorded at Wuhan station. In Fig. 5, the vertical profiles at $114^{\circ} \mathrm{E}$ and $30.5^{\circ} \mathrm{N}$ have been plotted. The comparisons show that the tomographically reconstructed density profiles agree well with those obtained from the IRI2001 model and the ionosonde data recorded at Wuhan station as a whole, but the profile of tomographic reconstruction is closer to that obtained from the ionosonde data, especially at the peak height. The excellent agreement between these independently measured data and the tomographically reconstructed density profiles, as shown in the examples in Fig. 5, confirms the capability of ionospheric tomography in monitoring the temporal and spatial variation of the ionospheric electron density.

\section{DISCUSSION AND CONCLUSION}

We have developed an HRA to fix the solution in the inverse problem of stochastic tomography. Such approaches have proved to be superior to TSVD or the ART alone in the numerical simulation experiment, yielding a more accurate and physical solution than the individual reconstruction algorithm. The method is then successfully applied to reconstruct the ionospheric electron density distribution with the GPS observations obtained from CMONOC. These results are beneficial for understanding the characteristics and variation mechanism of the ionosphere over China. Furthermore, they are capable of providing valuable experimental support for understanding the complex behavior of the low-latitude $\mathrm{F}$ region and for improving the existing ionospheric models such as the International Reference Ionosphere model.

Although the HRA usually takes slightly longer than the TSVD or the ART methods alone when they are applied to reconstruct the IED images, the time differential is negligible for high-performance computers. In the future, with the addition of a third GPS frequency and the application of the GALILEO system, the phase-leveling error should be significantly reduced and the carrier-phase noise level more closely approached. All these improvements will make us obtain more accurate TEC data. Thus, the main error source in the proposed HRA method is the discretization error, and the reconstruction technique should be further improved in order to decrease the impacts of the discretization error on the quality of the reconstructed tomography images. In addition, the main aim of this work is to get the 3-D image of the ionosphere by means of a tomographic technique. The temporal evolution of the IED is not considered in this work. In the future, this method would be extended to a fully $4 \mathrm{D}$ tomographic reconstruction by considering the temporal evolution of the ionosphere.

\section{ACKNOWLEDGMENT}

The ground-based GPS tracking station data were provided by the data center of the Crustal Movement Observation Network of China. The ionosonde data were provided by Prof. L. Liu with the Institute of Geology and Geophysics at the Chinese Academy of Sciences. Information System and Data Center (ISDC) GeoForschungsZentrum Potsdam (GFZ) provided the CHAllenging Minisatellite Payload (CHAMP) occultation measurements. Data on the instrumental bias were provided by Dr. X. Huo with the Institute of Geodesy and Geophysics at the Chinese Academy of Sciences.

\section{REFERENCES}

[1] J. R. Austen, S. J. Franke, and C. H. Liu, "Applications of computerized tomography techniques to ionospheric research," in Radio Beacon Contribution to the Study of Ionization and Dynamics of the Ionosphere and Corrections to Geodesy. Oulu, Finland: Ouluensis Univ., 1986, pp. 25-32.

[2] T. D. Raymund, J. R. Austen, and S. J. Franke, "Application of computerized tomography to the investigation of ionospheric structure," Radio Sci., vol. 25 , no. 5, pp. 771-789, Oct. 1990.

[3] H. Na and H. Lee, "Resolution analysis of tomographic reconstruction of electron density profiles in the ionosphere," Int. J. Imaging Syst. Technol., vol. 2, no. 3, pp. 209-218, Oct. 1990.

[4] E. J. Fremouw, J. A. Secan, and B. M. Howe, "Application of stochastic inverse theory to ionospheric tomography," Radio Sci., vol. 27, no. 5, pp. 721-732, Sep. 1992.

[5] V. E. Kunitsyn and E. D. Tereshchenko, Ionospheric Tomography. Berlin, Germany: Springer-Verlag, 2003.

[6] E. S. Andreeva, A. V. Galinov, and V. E. Kunitsyn, "Radio tomography reconstruction of ionostation dip in the plasma near the Earth," J. Exp. Theor. Phys. Lett., vol. 52, no. 2, pp. 145-148, Mar. 1990.

[7] S. E. Pryse and L. Kersley, "A preliminary experimental test of ionospheric tomography," J. Atmos. Terr. Phys., vol. 54, no. 7/8, pp. 10071023, Aug. 1992.

[8] X. F. Ma and T. Maruyama, "Three-dimensional ionospheric tomography using observation data of GPS ground receivers and ionosonde by neural network," J. Geophys. Res., vol. 110, no. A5, p. A05308, May 2005. DOI: 10.1029/2004JA010797.

[9] G. A. Hajj, R. Ibanez-Meier, E. R. Kursinski, and L. J. Roman, "Imaging the ionosphere with the global positioning system," Int. J. Imaging Syst. Technol., vol. 5, no. 2, pp. 174-184, Oct. 1994.

[10] A. J. Hansen, T. Walter, and P. Enge, "Ionospheric correction using tomography," in Proc. Inst. Navigat. GPS, 1997, pp. 249-257.

[11] A. Rius, G. Ruffini, and L. Cucurull, "Improving the vertical resolution of ionospheric tomography with GPS occultation," Geophys. Res. Lett., vol. 24, no. 18, pp. 2291-2294, Sep. 1998.

[12] D. B. Wen, Y. B. Yuan, J. K. Ou, X. L. Huo, and K. F. Zhang, "Threedimensional ionospheric tomography by an improved algebraic reconstruction technique," GPS Solut., vol. 11, no. 4, pp. 251-258, Nov. 2007. DOI: $10.1007 / \mathrm{s} 10291-007-0055-\mathrm{y}$.

[13] D. B. Wen, Y. B. Yuan, J. K. Ou, X. L. Huo, and K. F. Zhang, "Ionospheric temporal and spatial variations during the 18 August 2003 storm over China," Earth Planets Space, vol. 59, no. 4, pp. 313-317, May 2007.

[14] D. B. Wen, Y. B. Yuan, and J. K. Ou, "Monitoring the three-dimensional ionospheric electron distribution using GPS observations over China," J. Earth Syst. Sci., vol. 116, no. 3, pp. 235-244, Jun. 2007.

[15] M. Hernandez-Pajares, J. M. Juan, and J. Sanz, "Global observation of the ionospheric electronic response to solar events using ground and LEO GPS data," J. Geophys. Res., vol. 103, no. A9, pp. 20789-20796, Sep. 1998.

[16] M. Hernandez-Pajares, J. M. Juan, and J. Sanz, "Application of ionospheric tomography to real-time GPS carrier-phase ambiguities, at scales 400-1000 km and with high geomagnetic activity," Geophys. Res. Lett., vol. 27, no. 13, pp. 2009-2012, Jul. 2000. 
[17] G. Ruffini, A. Flores, and A. Rius, "GPS tomography of the ionospheric electron content with a correlation functional," IEEE Trans. Geosci. Remote Sens., vol. 36, no. 1, pp. 143-153, Jan. 1998.

[18] B. M. Howe, K. Runciman, and J. A. Secan, "Tomography of the ionosphere: Four-dimensional simulations," Radio Sci., vol. 33, no. 1, pp. 109-128, Jan./Feb. 1998.

[19] G. S. Bust, D. Coco, and J. J. Makela, "Combined ionospheric campaign 1: Ionospheric tomography and GPS total electron content (TEC) depletions," Geophys. Res. Lett., vol. 27, no. 18, pp. 2849-2852, Sep. 2000.

[20] C. N. Mitchell and P. S. Spencer, "A three-dimensional time-dependent algorithm for ionospheric imaging using GPS," Ann. Geophys., vol. 46, no. 4, pp. 687-696, Aug. 2003.

[21] C. Stolle, S. Schluter, and C. Jacobi, "3-dimensional IED reconstruction based on GPS measurements," Adv. Space Res., vol. 31, no. 8, pp. 19651970, Apr. 2003.

[22] P. Yin, C. N. Mitchell, and P. S. J. Spencer, "Ionospheric electron concentration imaging using GPS over the USA," Geophys. Res. Lett., vol. 31, no. 12, p. L12806, Jun. 2004. DOI: 10.1029/2004GL019899.

[23] P. C. Hansen, "The truncated SVD as a method for regularization," BIT, vol. 27, no. 4, pp. 534-553, Oct. 1987.

[24] M. Kunitake, K. Ohtaka, and T. Maruyama, "Tomographic imaging of the ionosphere over Japan by the modified truncated SVD method," Ann. Geophys., vol. 13, no. 12, pp. 1303-1310, Dec. 1995.

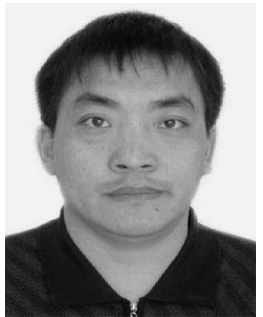

Debao Wen received the B.Sc. degree in surveying engineering from Henan Polytechnic University, Jiaozuo, China, and the Ph.D. degree in geodesy and surveying engineering in 2008 from the Chinese Academy of Sciences (CAS) Wuhan.

$\mathrm{He}$ is currently with the Institute of Geodesy and Geophysics, CAS, with the Changsha University of Science and Technology, Changsha, China, and with the Graduate School of CAS, Beijing. His current research projects include three-dimensional ionospheric/atmospheric tomography based on GNSS observations and the effects of space weather on navigation and positioning.

Dr. Wen has received several awards, including the Prize of Chinese Military Science and Technology Advance, Excellent Prize of the Presidential Scholarship of CAS, Student Best Paper of ION GNSS 2007.

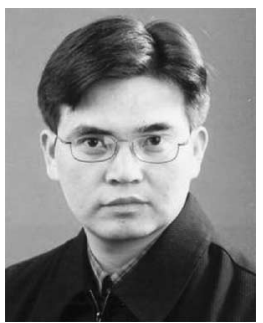

Yunbin Yuan received the BSc. degree in surveying engineering from the Shandong University of Science and Technology, Qingdao, China, in 1995 and the $\mathrm{Ph} . \mathrm{D}$. degree in geodesy and surveying in 2002 from the Institute of Geodesy and Geophysics, Chinese Academy of Sciences (CAS), Beijing.

His main subjects include satellite geodesy and GNSS positioning/atmosphere/ionosphere. His current research interests are GNSS-based spatial environmental monitoring and analysis, including the ionospheric correction for Network RTK for centimeter-level real-time positioning; high-precision GNSS satellite navigation and positioning in different augmentation systems such as WAAS and Network RTK; GNSS in applications to orbit determination for Low-Earthorbiter satellites and China's Manned's space-engineering.

Dr. Yuan has received several awards, including the Prize of Chinese Military Science and Technology Advance, Prize of Significant Contributions to China's Manned Spaceflight, China's Best PH.D thesis, Special Prize of the President Scholarship (CAS), and the 2001 ION GPS Student Best Paper.

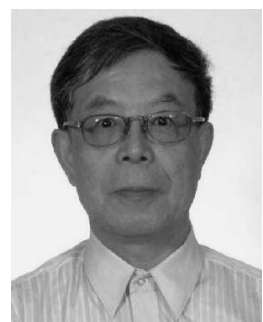

Jikun Ou received the B.Sc. degree in mechanical engineering in 1970 from the Beijing Machine College of China, Beijing, and the M.Sc. and Ph.D. degrees in geodesy from the Institute of Geodesy and Geophysics (IGG), Chinese Academy of Sciences, Beijing, in 1982 and 1994 respectively.

$\mathrm{He}$ is currently a Research Professor of IGG, and a doctoral supervisor of the graduate university of the Chinese Academy of Sciences. He is an editor for five journals. Since 1980 he has been performing research on the theory of errors in geodesy and surveying. He is the author of more than 100 scientific and technical research articles. He has obtained a special allowance from the State Council of China since 1993, and was chosen as a specialist of outstanding contributions in Hubei province. His current major areas of expertise include the control of data quality, detection of gross errors, precisely positioning by GPS, atmosphere and its effects on GPS surveying, and the approach of precise orbit determination for low orbital satellites and GNSS satellites, so on.

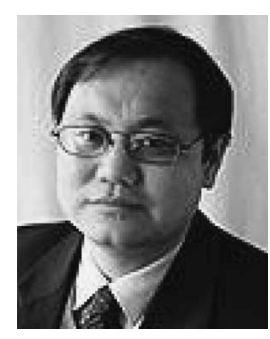

Kefei Zhang received the B.Sc. and M.Sc. degrees from Wuhan University (formerly Wuhan Technical University of Surveying and Mapping), Wuhan, China, in 1985 and 1988, respectively, and the Ph.D. degree from the Curtin University of Technology, Perth, Australia, in 1997, all in geodesy.

$\mathrm{He}$ is currently an Associate Professor and leads the Measurement Science Group at the School of Mathematical and Geospatial Sciences, Royal Melbourne Institute of Technology (RMIT), Melbourne, Australia. He has a mixed background of satellite positioning, tracking, and geodesy. Prior to joining RMIT in 1999 , he worked at the Institute of Engineering Surveying and Space Geodesy, University of Nottingham as a Postdoctoral Researcher for about three years. $\mathrm{He}$ is also a Guest Professor of a few international universities. His current research projects primarily involve algorithm development and innovative applications of GPS and GNSS technologies for high-accuracy positioning, atmospheric and meteorological studies, network-based RTK, and people and object tracking. In addition, he is also interested in geodesy-related theoretical and practical studies such as Earth's gravity field modelling, satellite orbit determination, and spectral and fractal analyses. He is the author of about 100 peer-reviewed publications in these fields since 1990.

Dr. Zhang is a member of a number of international special study groups and the editorial boards of scientific journals. He is the President of the International Association of Chinese Professionals in Global Positioning Systems.

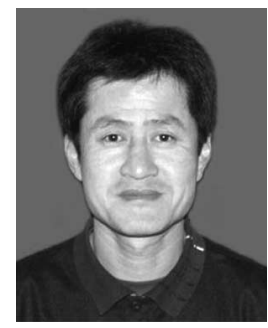

Kai Liu received the B.Sc. degree in surveying engineering from Shanghai Tongji University, Shanghai, China, in 1990 and the M.Sc. degree in geodesy and surveying from the Shandong University of Science and Technology, Qingdao, China, in 2005.

$\mathrm{He}$ is currently an Engineer in the Jinan Geotechnical Investigation and Surveying Institute, Jinan, China. His current research interests include the $3 \mathrm{~S}$ technique and the monitoring of crustal deformation based on GPS observations. 